\title{
Prevalence, risk factors, and the desire for help of distressed newly diagnosed cancer patients: A large-sample study*
}

GING-LONG WANG, M.D., M.P.H., F.A.A.C.A.P., ${ }^{1,2}$ CHIH-TAO CHENG, M.D., DR.P.H., ${ }^{1,3}$ AN-CHEN FENG, M.P.H., ${ }^{4}$ SHENG-HUI HSU, M.D., ${ }^{1}$ YI-CHEN HOU, M.s., ${ }^{1}$ AND CHIU-YUAN CHIU, Q.C.S.w. ${ }^{5}$

${ }^{1}$ Department of Psychiatry, Koo Foundation-Sun Yat-Sen Cancer Center, Taipei, Taiwan

${ }^{2}$ Department of Psychiatry, National Yang-Ming University School of Medicine, Taipei, Taiwan

${ }^{3}$ Department of Psychology and Social Work, National Defense University, Taipei, Taiwan

${ }^{4}$ Office of Epidemiology and Biostatistics, Koo Foundation-Sun Yat-Sen Cancer Center, Taipei, Taiwan

${ }^{5}$ Department of Social Work, Koo Foundation-Sun Yat-Sen Cancer Center, Taipei, Taiwan

(Received June 30, 2015; Accepted August 16, 2016)

\begin{abstract}
Objective: Beginning in 2007, all newly diagnosed cancer patients at the Koo Foundation Sun Yat-Sen Cancer Center (KF-SYSCC) were screened for psychosocial distress. Our social workers, as part of the psychosocial care team (PCT), have engaged in proactive outreach with patients identified as distressed. The goal of the present study was to assess the prevalence of psychosocial distress and the extent of contact between the PCT and distressed patients.

Method: Newly diagnosed patients who were treated at KF-SYSCC between 2007 and 2010 for cancer were eligible if there were at least 100 patients with the same type of cancer. Before treatment began, they were screened with the Pain Scale and the Distress Thermometer (DT) and had the option to specify a desire for help. The rates of distress were analyzed by cancer type and by probable related factors. Information regarding contact with the PCT was retrieved from computerized databases.

Results: Overall, some 5,335 cancer patients representing 12 major cancer types were included in our study. Of these, 1,771 (33.20\%) were significantly distressed. By multivariate logistic regression, younger age, female gender, higher pain score, and disease stage, but not cancer type, were found to be associated with higher rates of distress. Among these distressed patients, $628(36 \%)$ had some contact with the PCT.

Significance of results: This Taiwanese study with a large sample size revealed a prevalence rate of psychosocial distress similar to rates found in Western countries. Contact with the PCT was established in only $36 \%$ of significantly distressed patients, despite a proactive outreach program. It is very important to have screening results made available in a timely fashion to the psycho-oncology team so that appropriate care can be offered promptly.
\end{abstract}

KEYWORDS: Distress, Risk factors, Desire for help, Psycho-Oncology, Psychosocial care team

\footnotetext{
*Parts of this paper were presented as posters at the 2012 IPOS Meeting in Brisbane, Australia.

Address correspondence and reprint requests to: Ging-Long Wang, Department of Psychiatry, Koo Foundation Sun Yat-Sen Cancer Center, 125 Lih-Der Road, Bei-Tou District, Taipei, Taiwan 11259. E-mail: glw@kfsyscc.org.
}

\section{INTRODUCTION}

It has been well documented that the rates of psychosocial distress in cancer patients at different stages of their treatment are high and that psychosocial interventions can be effective (NCCN, 2003; Kissane et al., 2007; Institute of Medicine, 2008; Watson \& Kissane 2011; Moorey \& Greer, 2012; Moorey, 
2013). The prevalence of distress has been estimated to be between 25 and $47 \%$ in Western countries (Derogatis et al., 1983; Farber et al., 1984; Stefanek et al., 1987; Zabora et al., 2001; Carlson et al., 2004; Kendall et al., 2011; Dolbeault et al., 2011; Gunnarsdottir et al., 2012; Chambers et al., 2014). Distress prevalence in Asian countries has been reported to range from 30 to $50 \%$ (Uchitomi et al., 2003; Akechi et al., 2004; Akizuki et al., 2005; Lam et al., 2007; Shim et al., 2008; Wang G et al., 2011; Wang Y et al., 2013). The difference in prevalence rates may be related to the screening tools employed and the populations involved. The sample sizes in most of these studies were relatively small-between 100 and 450. There were a few exceptions. The first was the landmark study by Zabora and colleagues (2001), who utilized the 53-item Brief Symptom Inventory (BSI) on newly diagnosed cancer patients at an oncology center in the United States with a sample size of 4,496 over a period of 6 years. The overall prevalence rate of distress for newly diagnosed cancer patients was found to be $35.1 \%$, with a range of $29.6-43.4 \%$. Three other studies utilizing the BSI-18 or the Distress Thermometer (DT) on more than a thousand patients reported a distress prevalence rate of 32-44\% (Carlson et al., 2004; Kendall et al., 2011; Grassi et al., 2013).

Many of these studies demonstrated that gender, age, types of cancer, stage of diseases, pain, physical problems, social support, past psychiatric history, and financial stress are risk factors for psychosocial distress (Chen et al., 2000; Zabora et al., 2001; Carlson et al., 2004; Grassi et al., 2013; Sharp et al., 2013).

Despite strong recommendations from the National Comprehensive Cancer Network (NCCN) and Institute of Medicine, routine screening for distress has not been offered in the majority of cancer care organizations in the U.S. (Deshields et al., 2013). After the distress screening, most distressed patients elected not to seek help (Carlson et al., 2004). The levels of desire for help with distress were found to be low between $12 \%$ and $36 \%$ (Söllner et al., 2004; Graves et al., 2007; Baker-Glenn et al., 2011; Clover et al., 2013; Dubruille et al., 2015). The rates of referral acceptance by the distressed patients were found to be even lower: most of the studies reported rates between $17.8 \%$ and $28.2 \%$ (Shimizu et al., 2005; Shimizu et al., 2010; Ito et al., 2011; Bauwens et al., 2014). However, the distress screening was found to contribute to the earlier start of psychiatric treatment (Ito et al., 2011). Routine screening for distress followed by personalized triage also resulted in more benefits for patients (Carlson et al., 2013). In order to facilitate referral acceptance, it has been recommended that patients should be asked whether they want to be referred for additional psychosocial care (Tuinman et al., 2008; Ryan et al., 2012; Bauwens et al., 2014).

The DT, with acceptable validity, has been recommended by the NCCN for screening of psychosocial distress (NCCN 2003; Jacobson et al., 2005; Bultz et al., 2006; Holland et al., 2011). It has been widely utilized internationally (Khatib et al., 2004; Jacobson et al., 2005; Akizuki et al., 2005; Ozalp et al., 2007; Shim et al., 2008; Lam et al., 2007; Holland et al., 2011; Wang et al., 2011; Dolbeault et al., 2011; Carlson et al., 2012; Gunnarsdottir et al., 2012; Wang et al., 2013). At this Cancer Center (KF-SYSCC), the DT, including the Problem List (PL), has been used to screen distress for all new outpatients since 2007. When patients are identified as significantly distressed and express the desire for help from our Psychosocial Care Team (PCT), our social workers would initiate telephone or personal contact with them. When needed, patients were referred for further appropriate assessment and treatment. The PCT consists of 8 social workers, 1 clinical psychologist and 3 psychiatrists. Other resources include 2 spiritual counselors and 95 volunteers, who provide individual and/or group support.

The goal of this study was to assess, in a sizable sample, the prevalence of psychosocial distress among different cancer types and the probable related factors. We also retrospectively assessed the extent of contact established between the distressed patients and the PCT. We hypothesized that the prevalence of distress would be similar to that reported in Western countries; ethnic and cultural elements might affect the risk factors and psychosocial care patterns; and rates of contact with the psychosocial care team might be higher than reported by previous studies because of the initial outreach by our social workers.

\section{METHODS}

\section{Data Collection}

We retrieved data from the health information system at KF-SYSCC. Our cancer registry provides information about gender, age, cancer diagnosis, cancer site, cancer stage, date of cancer diagnosis, and cancer treatment received. Information on contact with the psychosocial care team (PCT)—including psychiatric consultations, outpatient visits, and psychologist visits-was obtained from registry data. There is a hospital information system at our cancer center that provides computerized physician order entry, imaging reports, laboratory and pathology reports, surgical records, and outpatient physician notes. The DT and pain scores were collected from this system. 


\section{Study Population and Eligibility}

Data on all newly diagnosed cancer patients treated at KF-SYSCC between 2007 and 2010 who fit the following criteria were retrospectively collected for our study:

1. A cancer diagnostic category that had a sample size $\geq 100$ in our study period (a total of 12 cancer types were selected).

2. Age 18 or older.

3. DT screening performed within 90 days of the diagnosis and before any treatment for that diagnosis.

Patients were approached by the nursing staff at the outpatient clinic to complete the Pain Scale and the DT/PL screening, along with an additional yes-andno question: "Would you like to receive help from our psychosocial care team?" The questionnaires were collected at the outpatient clinic or sent in by mail from patients to the Department of Social Services.

\section{Screening Tools/Questionnaires}

DT screening is a self-report questionnaire that gathers information on the level of a patient's distress during the past 7 days on a scale of 0 to 10 , with $0=$ no distress and $10=$ extreme distress. It includes a Problem List, which consists of 39 questions about physical, practical, family, emotional, and spiritual/religious problems. The cutoff score for DT screening was set at $\geq 4$, based on a previous study at our cancer center (Wang et al., 2011). The pain score also used a $0-10$ scale, with $0=$ no pain and $10=$ unbearable pain. According to previous studies (Tanaka et al., 2002; Gerbershagen et al., 2011), a pain score $\geq 4$ is considered moderate, which nevertheless requires intervention.

\section{Statistical Analysis}

We conducted a descriptive analysis of the demographic variables (gender, age) of the sample and the timing of screening. Rates of distress (percentage with a DT score $\geq 4$ ) were summarized according to cancer type and such probable related factors as gender, age, disease stage, and pain score. In the univariate analyses, Pearson chi-square tests and an unadjusted $p$ value were employed to demonstrate associations between prevalence rates of distress and categorical risk factors (gender, age, cancer type, cancer stage, and pain score). A stepwise logistic regression was also performed to fit the final model of distress, and adjusted $p$ values were obtained. Information regarding patient contact with the PCT was retrieved from the computerized databases. We calculated the number of patients contacted by the PCT and plotted the timing of these contacts after DT screening. A value of $p<0.05$ was considered to be statistically significant in all our analyses.

\section{Ethical Considerations}

The institutional review board at KF-SYSCC approved analysis of these data as a quality-improvement evaluation of clinical services and thus waived the requirement for obtaining informed consent from individual patients.

\section{RESULTS}

From the first day of 2007 to the last day of 2010, there were 5,770 newly diagnosed cancer patients seen at the outpatient clinic who had fellow-up oncology treatment at our cancer center: 8 were excluded for being younger than 18, and 427 were excluded because the number of cases for their cancer type did not reach the inclusion criteria ( $\geq 100$ cases). Therefore, 5,335 newly diagnosed cancer patients representing 12 major cancer types were included in our study. Some $61.5 \%$ of our sample were women, which directly correlates with the breast cancer program, the largest program at our cancer center. Patient ages ranged from 18 to 96 years, with a mean of 53 . The duration between cancer diagnosis and DT assessment ranged between 0 and 90 days (mean $=14.1$ days, $S D=13.6$ days). The 10 th and 90 th percentiles for duration were 0 and 30 days, respectively (Table 1 ).

Overall, 1,771 (33.20\%) patients were found to be significantly distressed, with a DT score $\geq 4$. The prevalence rate varied from 22 to $36 \%$ among cancer types. Esophageal, breast, nasopharyngeal, gastric, thyroid, and lung cancers showed higher rates, while prostate and bladder cancer had the lowest. The differences were moderate (Figure 1).

Pearson chi-square tests for associations revealed that female gender, younger age, and higher pain score were associated with higher rates of distress, while stage of disease was not. However, when the stepwise logistic regression was performed, the final model identified four variables that met the $p<0.05$ significance level for entry into the model: female gender, younger age $(<53$ years $)$, pain score $\geq 4$, and stage 4 disease were statistically associated with distress (by adjusted $p$ value, Table 2).

Among the 1,771 significantly distressed patients who were willing to receive assistance from the PCT, 628 (36\%) established contact: 352 (20\%) with only social workers, 206 (12\%) with only psychiatrists, and $70(4 \%)$ with both social workers and psychiatrists. As a result, of all the significantly distressed patients, $422(24 \%)$ had contact with social workers and 276 (16\%) with psychiatrists (Figure 2). 
Table 1. Gender, age, and timing of DT assessment for the prevalence sample $(\mathrm{N}=5,335)$

\begin{tabular}{ll}
\hline \hline Gender & \\
Female & $3,282(61.5 \%)$ \\
Male & $2,053(38.5 \%)$ \\
Age & \\
Mean & 53 \\
Range & $18-96$ \\
Days after cancer diagnosis & \\
$\quad$ (when DT was assessed) & 14 \\
Mean & $0-90$ \\
Range & \\
\hline
\end{tabular}

The vast majority of these patients (up to 86\%) had contact with social workers within 30 days of screening, as the social workers had made the initial effort to reach out to most of them. Visits to psychiatric staff were spread out over a year, with only $52 \%$ within the first three months (Figure 3).

\section{DISCUSSION}

\section{Prevalence of Distress and Associated Factors}

Our study revealed that between 22 and $36 \%$ of newly diagnosed cancer patients are significantly distressed.
As we had hypothesized, these results are similar to those reported in Western countries (Zabora et al., 2001; Carlson et al., 2004; 2012). There are moderate differences in prevalence rates among different cancer types. The rate of significant distress was positively associated with younger age, female gender, and higher pain score, and somewhat with stage of disease. The weak association between disease stage and rate of distress may be partially due to cultural factors and family relationships. It has been observed that a good portion of the Asian patient population may be informed only of their diagnosis and not of the stage of their illness, or their prognosis. Usually, only family members are fully apprised of a patient's condition (Tang \& Lee, 2004; Li et al., 2012). This cultural factor associated with truthtelling practices will affect a patient's subsequent emotional response to distress.

Our univariate analysis showed that the prevalence of distress varies from one cancer type to another. However, the multivariate logistic regression conducted on type III analysis of effects based on the Wald test showed that cancer type was not statistically significant $(p=0.20)$. This indicates that, after controlling for gender, age, disease stage, and pain score, cancer type was not associated with a difference in prevalence of distress. The difference in DT score by cancer type seen in the univariate

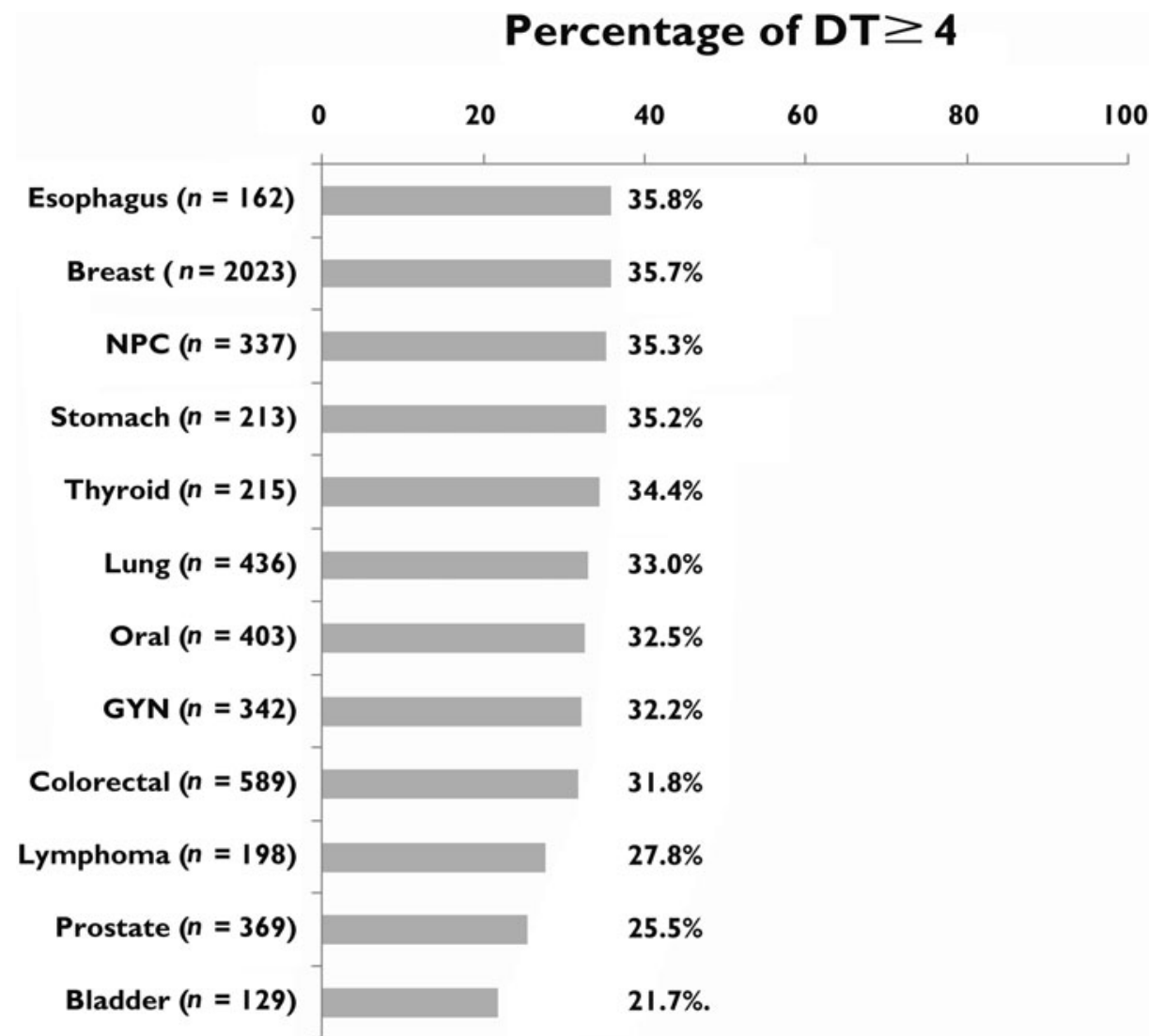

Fig. 1. Prevalence of distress (DT $\geq 4)$ and number of patients by cancer type $(N=5,335)$. 
Table 2. Prevalence rates of distress for five categorical risk factors $(\mathrm{N}=5,335)$

\begin{tabular}{|c|c|c|c|c|c|}
\hline & Number of patients & $\mathrm{DT} \geq 4$ & $\mathrm{DT}<4$ & Unadjusted $p$ value $^{\mathrm{a}}$ & Adjusted $p$ value ${ }^{\mathrm{b}}$ \\
\hline All patients & $5335(100 \%)$ & $1770(33.2 \%)$ & $3565(66.8 \%)$ & & \\
\hline Gender & & & & $<0.001$ & $<0.001$ \\
\hline Male & $2053(38.5 \%)$ & $589(28.7 \%)$ & $1464(71.3 \%)$ & & \\
\hline Female & $3282(61.5 \%)$ & $1181(36.0 \%)$ & $2101(64.0 \%)$ & & \\
\hline Age & & & & $<0.001$ & $<0.001$ \\
\hline$<53$ & $2626(49.2 \%)$ & $985(37.5 \%)$ & $1641(62.5 \%)$ & & \\
\hline$>53$ & $2709(50.8 \%)$ & $785(29.0 \%)$ & $1924(71.0 \%)$ & & \\
\hline Stage 4 disease & & & & 0.13 & 0.049 \\
\hline No & $4202(78.8 \%)$ & $1373(32.7 \%)$ & $2829(67.3 \%)$ & & \\
\hline Yes & $1133(21.2 \%)$ & $397(35.0 \%)$ & $736(65.0 \%)$ & & \\
\hline Pain score & & & & $<0.001$ & $<0.001$ \\
\hline$<4$ & $4832(90.6 \%)$ & $1522(31.5 \%)$ & $3310(68.5 \%)$ & & \\
\hline$\geq 4$ & $503(9.4 \%)$ & $248(49.3 \%)$ & $255(50.7 \%)$ & & \\
\hline Cancer type & & & & 0.003 & \\
\hline Breast & $2032(38.1 \%)$ & $726(35.7 \%)$ & $1306(64.3 \%)$ & & \\
\hline Colorectal & $589(11.0 \%)$ & $187(31.8 \%)$ & $402(68.3 \%)$ & & \\
\hline Lung & $436(8.2 \%)$ & $144(33.0 \%)$ & $292(67.0 \%)$ & & \\
\hline Oral & $403(7.6 \%)$ & $131(32.5 \%)$ & $272(67.5 \%)$ & & \\
\hline Prostate & $369(6.9 \%)$ & $94(25.5 \%)$ & $275(74.5 \%)$ & & \\
\hline Gynecological & $342(6.4 \%)$ & $110(32.2 \%)$ & $232(67.8 \%)$ & & \\
\hline NPC & $337(6.3 \%)$ & $119(35.3 \%)$ & $218(64.7 \%)$ & & \\
\hline Stomach & $213(4.0 \%)$ & $75(35.2 \%)$ & $138(64.8 \%)$ & & \\
\hline Lymphoma & $198(3.7 \%)$ & $55(27.8 \%)$ & $143(72.2 \%)$ & & \\
\hline Esophagus & $162(3.0 \%)$ & $58(35.8 \%)$ & $104(64.2 \%)$ & & \\
\hline Bladder & $129(2.4 \%)$ & $28(21.7 \%)$ & $101(78.3 \%)$ & & \\
\hline Thyroid & $125(2.3 \%)$ & $43(34.4 \%)$ & $82(65.6 \%)$ & & \\
\hline
\end{tabular}

${ }^{a}$ Unadjusted $p$ value: chi-squared test.

${ }^{\mathrm{b}}$ Adjusted $p$ value: logistic regression. 

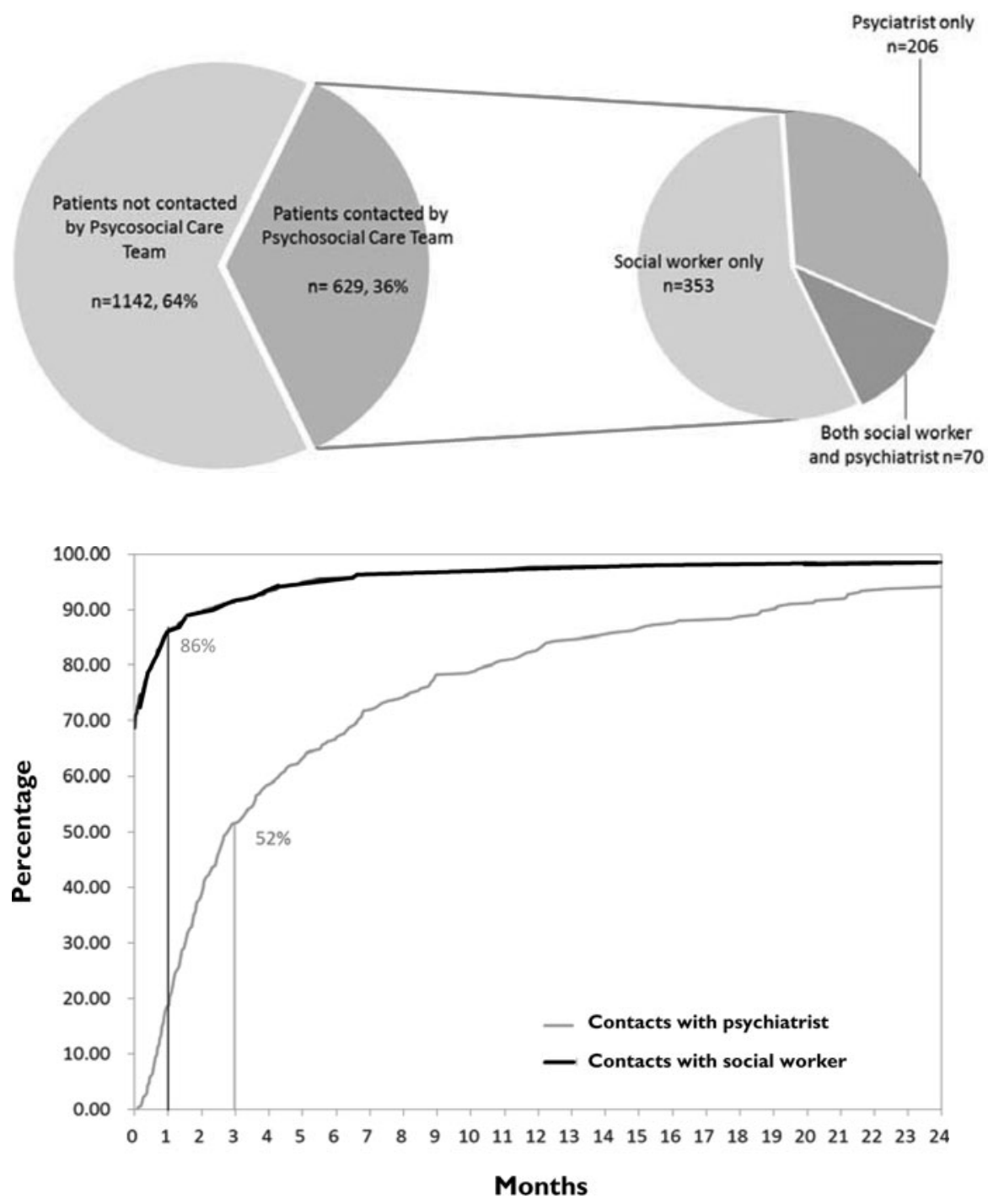

Fig. 2. Contacts with the psychosocial care team by patients with $\mathrm{DT} \geq 4$. analysis can be accounted for by differences in gender, age, disease stage, and pain score.

\section{Contacts with the PCT}

In our study, the PCT established contact with up to $36 \%$ of distressed patients, which exceeds the higher range of most of previously reported rates of referral acceptance of 17.8-28.2\% (Shimizu et al., 2005; 2010; Ito et al., 2011; Bauwens et al., 2014). One exception was a study that reported an acceptance rate of $46.8 \%$ in 284 distressed patients referred to a psycho-oncology service by oncology staff (Grassi et al., 2011). In that study, the staff were first trained through seminars, including webcast lectures, on the psychosocial aspects of cancer care, emotional distress, and the use of the DT/PL. This may have contributed to the much higher referral acceptance rate. However, there are reviews that discourage confidence in significant changes in clinical practice being delivered by simple educational interventions
(O'Brien et al., 2007; Forsetlund et al., 2009; Bauwens et al., 2014).

Our results with a higher rate of contact with the PCT are partly related to the fact that our social workers proactively made phone contacts with distressed patients who had expressed a willingness to receive assistance from the team. After the initial phone call, social workers would arrange for personal or phone interviews. If necessary, patients could be referred to a psychiatrist, a clinical psychologist, or a spiritual counselor for further care. However, there was a small percentage of distressed patients who requested a meeting with social workers through referrals from the attending oncologists or due to their need for assistance with financial or other practical problems. This type of contact with social workers was estimated to be below $5 \%$ of the $24 \%$ of distressed patients who had contact with social workers.

In terms of psychiatric contacts, our finding of 16\% is comparable to two previous studies conducted in Japan (Shimizu et al., 2010; Ito et al., 2011). Ito 
and colleagues (2011) reported that, of 520 patients who started chemotherapy during a 6 -month period, $26(5 \%)$ were seen for psychiatric assessment and treatment. The number of patients screened positive for distress was 146 (of a total of 441 screened), yielding a distress rate of $33.1 \%$. All patients who screened positive were recommended for a consultation with psychiatric services. Therefore, the referral acceptance rate was $17.8 \%(26 / 146)$. In another study, Shimizu and coworkers (2010) reported that $25 \%$ of distressed patients received psychiatric visits. This higher rate appeared to be related to the fact that they had made every effort to schedule psychiatric appointments on the same day as the screening.

It is worth noting that contact with social workers occurred mostly within 30 days of their diagnosis, but only half of the visits to psychiatrists took place within the first 3 months. This may be due to the fact that most of patients were preoccupied with the immediate need for medical and physical management of their illness. Seeking psychiatric/psychological help can be stigmatic, or it may not be at the top of a distressed patient's priority list. Many distressed patients may prefer self-help, may fail to consider their problems serious, or are already in therapy for their distress (Wang et al., 2009; Clover et al., 2013; 2015). Our patients were screened weekly during all their hospital stays (for surgery or other treatments). If repeated screening showed significant distress, it could lead to further psychiatric referrals, which could be better accepted as the processes of medical treatment evolved. This may also explain why visits to the psychiatric staff were spread out in the months following their cancer diagnosis.

\section{STRENGTHS AND LIMITATIONS}

One of the strengths of our study is its large sample size. The sample sizes of most previous studies on distress have ranged between 100 and 450 . There were four studies that had sample sizes greater than 1,000. The first was reported by Zabora et al. (2001), with a sample size of 4,496 . The second study used the BSI-18 on all outpatients at all treatment stages at a tertiary cancer center in Canada and had a sample size of 2,276 over a period of 4 weeks. They reported that $37.8 \%$ of their cancer patients were distressed (Carlson et al., 2004). The third and fourth studies utilized the DT to assess cancer outpatients. The third screened outpatients during their first visit to the medical/radiation oncology clinic at a community cancer center in the United States. Of the 1,281 screenings collected over a period of 9 months, 32\% rated distress above the threshold (DT $\geq 4$ ) (Kendall et al., 2011). The fourth, a multi- center nationwide study in Italy conducted during an index week at all centers, screened 1,108 newly diagnosed outpatients with cancer over a 2-day period. Some $47 \%$ of these patients fulfilled the criteria for distress when the DT cutoff was set at $\geq 4$, and $33 \%$ when the cutoff was $\geq 5$ (Grassi et al., 2013).

The weakness of our study is that it is retrospective in nature. The sample consisted only of patients who chose to start treatment at our cancer center, which amounted to about $60 \%$ of all new cancer outpatients. Up to $40 \%$ of the patients in our study had already received a cancer diagnosis or had been informed that they might have cancer when they first came to the clinic. Some may have gone "hospital shopping," thus delaying initiation of treatment, as about $10 \%$ of the sample was screened more than 30 days after their diagnosis. Another weakness is that this is a single-institution study. Caution is required when generalizing our results to other oncology settings.

\section{CLINICAL IMPLICATIONS}

Our rate of contact with the PCT by distressed patients is relatively high among this type of study, but it could be even higher if it weren't for the low return rate of questionnaires, as many were not completed immediately during outpatient clinic visits. This led us to initiate a new program using electronic devices where patients could complete questionnaires on a touch screen, so that the results could be made available to the staff on a real-time basis, which will hopefully lead to prompt and appropriate delivery of psychosocial care for distressed patients.

\section{CONCLUSIONS}

Screening for psychosocial distress in newly diagnosed cancer patients at KF-SYSCC revealed that as many as a third of patients were significantly distressed, with up to $36 \%$ identified as distressed making contact with the PCT. Most patient contacts with social workers occurred within a month of screening. However, only half of the initial psychiatric visits were made within the first three months. Engaging family members while assessing patients would be a very important approach for Asian patients, because the family plays such a key role in the patient's perception of and ability to cope with their illness. A patient's follow-up contact with the psychosocial care team, especially with the psychiatric staff, should be closely monitored to facilitate timely intervention. Further studies are needed to evaluate the outcome of routine screening of psychosocial distress, of follow-up assessments, and of interventions. 


\section{ACKNOWLEDGMENTS}

We wish to acknowledge the excellent editorial assistance rendered by Jamie Chen-Fenner and Mona Wang Ono.

\section{REFERENCES}

Akechi, T., Okuyama, T., Sugawara, Y., et al. (2004). Major depression, adjustment disorders, and post-traumatic stress disorder in terminally ill cancer patients: Associated and predictive factors. Journal of Clinical Oncology, 22(10), 1957-1965.

Akizuki, N., Yamawaki, S., Akechi, T., et al. (2005). Development of an Impact Thermometer for use in combination with the Distress Thermometer as a brief screening tool for adjustment disorders and/or major depression in cancer patients. Journal of Pain and Symptom Management, 29, 91-99. Available from http:// www.jpsmjournal.com/article/S0885-3924(04)00450-6/ abstract.

Baker-Glenn, E.A., Park, B., Granger, L., et al. (2011). Desire for psychological support in cancer patients with depression or distress: Validation of a simple help question. Psycho-Oncology, 20, 525-531. Available from http://onlinelibrary.wiley.com/doi/10.1002/pon. v20.5/issuetoc.

Bauwens, S., Baillon, C. \& Distelmans, W. (2014). Systematic screening for distress in oncology practice using the Distress Barometer: The impact on referrals to psychosocial care. Psycho-Oncology, 23(7), 804-811. Epub ahead of print Jan 24. Available from http://onlinelibrary.wiley.com/doi/10.1002/pon.3484/full.

Bultz, B. \& Carlson, L.E. (2006). Emotional distress: The sixth vital sign-future directions in cancer care. Psycho-Oncology, 15, 93-95. Available from http://onlinelibrary.wiley.com/doi/10.1002/pon.1022/pdf.

Carlson, L.E., Angen, M., Cullum, J., et al. (2004). High levels of untreated distress and fatigue in cancer patients. British Journal of Cancer, 90(12), 2297-2304.

Carlson, L.E., Waller, A. \& Mitchell, A.J. (2012). Screening for distress and unmet needs in patients with cancer: Review and recommendations. Journal of Clinical Oncology, 30(11), 1160-1177. Epub ahead of print Mar 12. Available from http://jco.ascopubs.org/content/early/2012/03/06/JCO.2011.39.5509.full.pdf.

Carlson, L.E., Waller, A., Groff, S.L., et al. (2013). Screening for distress, the sixth vital sign, in lung cancer patients: Effects on pain, fatigue, and common problems. Secondary outcomes of a randomized controlled trial. Psycho-Oncology, 22(8), 1880-1888. Epub ahead of print Nov 12, 2012. Available from http://onlinelibrary.wiley.com/wol1/doi/10.1002/pon.3223/full.

Chambers, S., Zajdlewicz, L., Youlden, D.R., et al. (2014). The validity of the Distress Thermometer in prostate cancer populations. Psycho-Oncology, 23(2), 195-203. Epub ahead of print Sep 12, 2013. Available from http://onlinelibrary.wiley.com/doi/10.1002/pon.3391/pdf.

Chen, M.L., Chang, H.K. \& Yeh, C.H. (2000). Anxiety and depression in Taiwanese cancer patients with and without pain. Journal of Advanced Nursing, 32(4), 944-951. Available from https://www.researchgate. net/publication/12233140_Anxiety_and_depression_ in_Taiwanese_cancer_patient_with_and_without_pain.

Clover, K., Kelly, P., Rogers, K., et al. (2013). Predictors of desire for help in oncology outpatients reporting pain or distress. Psycho-Oncology, 22(7), 1611-1617. Epub
Sep 27, 2012. Available from http://onlinelibrary.wiley. com/wol1/doi/10.1002/pon.3188/full.

Clover, K., Mitchell, A.J., Britton, B., et al. (2015). Why do oncology outpatients who report emotional distress decline help? Psycho-Oncology, 24(7), 812-818. Epub ahead of print Dec 11, 2014. Available from http://onlinelibrary.wiley.com/doi/10.1002/pon.v24.7/issuetoc.

Derogatis, L.R., Morrow, G.R., Fetting, J., et al. (1983). The prevalence of psychiatric disorders among cancer patients. The Journal of the American Medical Association, 249(6), 751-757. Available from http://jama. jamanetwork.com/article.aspx?articleid=383328/

Deshields, T., Zebrack, B. \& Kennedy, V. (2013). The state of psychosocial services in cancer care in the United States. Psycho-Oncology, 22(3), 699-703. Epub ahead of print Feb 21, 2012. Available from http://onlinelibrary.wiley.com/doi/10.1002/pon.3057/full.

Dolbeault, S., Boistard, B., Meuric, J., et al. (2011). Screening for distress and supportive care needs during the initial phase of the care process: A qualitative description of a clinical pilot experiment in a French cancer center. Psycho-Oncology, 20(6), 585-593. Epub ahead of print Mar 22. Available from http://onlinelibrary.wiley.com/ doi/10.1002/pon.1946/full.

Dubruille, S., Libert, Y., Merckaert, I., et al. (2015). The prevalence and implications of elderly inpatients' desire for formal psychological help at the start of cancer treatment. Psycho-Oncology, 24(3), 294-301. Epub ahead of print Jul 31, 2014. Available from http://onlinelibrary. wiley.com/doi/10.1002/pon.v24.3/issuetoc.

Farber, J.M., Weinerman, B.H. \& Kuypers, J.A. (1984). Psychosocial distress of oncology patients. Journal of Psychosocial Oncology, 2, 109-118. Available from http://onlinelibrary.wiley.com/doi/10.1002/pon.1085/pdf.

Forsetlund, L., Bjorndal, A., Richidian, A., et al. (2009). Continuing education meetings and workshops: Effects on professional practice and health care outcomes. The Cochrane Database of Systematic Reviews, 2, CD003030. Available from http://onlinelibrary.wiley. com/doi/10.1002/14651858.CD003030.pub2/abstract.

Gerbershagen, H.J., Rothaug, J., Kalkman, C.J., et al., (2011). Determination of moderate-to-severe postoperative pain on the numeric rating scale: A cut-off point analysis applying four different methods. British Journal of Anaesthesia, 107(4), 619-626. Epub ahead of print Jun 30. Available from http://bja.oxfordjournals. org/content/107/4/619.full.pdf+html.

Grassi, L., Rossi, L., Caruso, R., et al. (2011). Educational intervention in cancer outpatient clinics on routine screening for emotional distress: An observational study. Psycho-Oncology, 20(6), 669-674. Epub ahead of print Mar 2. Available from http://onlinelibrary.wiley. com/doi/10.1002/pon.1944/pdf.

Grassi, L., Johansen, C., Annunziata, M.A., et al. (2013). Screening for distress in cancer patients: A multicenter, nationwide study in Italy. Cancer, 119(9), 1714-1721. Epub ahead of print Feb 19. Available from http://onlinelibrary.wiley.com/doi/10.1002/cncr.27902/pdf.

Graves, K.D., Arnold, S.M., Love, C.L., et al. (2007). Distress screening in a multidisciplinary lung cancer clinic: Prevalence and predictors of clinically significant distress. Lung Cancer, 55, 215-224. Epub ahead of print Nov 3, 2006.

Gunnarsdottir, S., Thorvaldsdottir, G.H., Fridriksdottir, N., et al. (2012). The psychometric properties of the Icelandic version of the Distress Thermometer and Problem List. Psycho-Oncology (7), 21, 730-736. Epub 
ahead of print Mar 29, 2011. Available from http://onlinelibrary.wiley.com/wol1/doi/10.1002/pon.1950/full.

Holland, J.C. \& Bultz, B.D. (2011). The NCCN guideline for distress management: A case for making distress the sixth vital sign. Journal of the National Comprehensive Cancer Network, 5(1), 3-7. Available from http://www. pubfacts.com/detail/17323529/The-NCCN-guidelinefor-distress-management-a-case-for-making-distressthe-sixth-vital-sign.

Institute of Medicine (2008). Cancer care for the whole person: Meeting psychosocial health needs. Washington, D.C.: The National Academies Press.

Ito, T., Shimizu, K., Ichida, Y., et al. (2011). Usefulness of pharmacist-assisted screening and psychiatric referral program for outpatients with cancer undergoing chemotherapy. Psycho-Oncology, 20(6), 647-654. Epub ahead of print Mar 7. Available from http://onlinelibrary. wiley.com/doi/10.1002/pon.v20.6/issuetoc.

Jacobson, P.B., Donovan, K.A., Trask, P.C., et al. (2005). Screening for psychologic distress in ambulatory cancer patients: A multicenter evaluation of the Distress Thermometer. Cancer, 103(7), 1494-1502. Available from http://www.capo.ca/pdf/Jacobsen_et_al_2005.pdf.

Kendall, J., Glaze, K., Oakland, S., et al. (2011). What do 1281 distress screeners tell us about cancer patients in a community cancer center? Psycho-Oncology, 20(6), 594-600. Epub ahead of print Feb 8. Available from http://onlinelibrary.wiley.com/doi/10.1002/pon.1907/ pdf.

Khatib, J., Salhi, R. \& Awad, G. (2004). Distress in cancer in-patients in King Hussein Cancer Center (KHCC): A study using the Arabic-modified version of the Distress Thermometer. Psycho-Oncology, 13(Suppl. 1), S42-S48.

Kissane, D.W., Grabsch, B., Clarke, D.M., et al. (2007). Supportive-expressive group therapy for patients with primary breast cancer: Survival and psychosocial outcomes from a randomized controlled trial. Psycho-Oncology, 16(4), 277-286. Available from http://onlinelibrary. wiley.com/doi/10.1002/pon.1185/pdf.

Lam, W.W.T., Chan, M., Hung, W.K., et al. (2007). Treatment decision difficulties and post-operative distress predict persistence of psychological morbidity in Chinese women following breast cancer surgery. PsychoOncology, 16(10), 904-912. Available from http://onlinelibrary.wiley.com/doi/10.1002/pon.1147/pdf.

Li, J., Yuan, X.L., Gao, X.H., et al. (2012). Whether, when, and who to disclose bad news to patients with cancer: A survey in 150 pairs of hospitalized patients with cancer and family members in China. Psycho-Oncology, 21(7), 778-784. Epub ahead of print Apr 20, 2011. Available from http://onlinelibrary.wiley.com/wol1/doi/10.1002/ pon.1979/full.

Moorey, S. (2013). "I know they are distressed. What do I do now?" Psycho-Oncology, 22(9), 1946-1952. Epub ahead of print Jul 1. Available from http://onlinelibrary.wiley.com/doi/10.1002/pon.3297/full.

Moorey, S. \& Greer, S. (2012). The Oxford guide to CBT for people with cancer. Oxford: Oxford University Press.

National Comprehensive Cancer Network (NCCN) (2003). NCCN practice guidelines in oncology, Vol. 1: Distress management. Fort Washington, PA: National Comprehensive Cancer Network. Available from https://www. nccn.org/professionals/physician_gls/f_guidelines.asp.

O'Brien, M.A., Rogers, S. \& Jamtvedt, G. (2007). Educational outreach visits: Effects on professional and health care outcomes. The Cochrane Database of Systematic Reviews, 4, CD000409. Available from http://onlinelibrary.
wiley.com/wol1/doi/10.1002/14651858.CD000409.pub2/ full.

Ozalp, E., Cankurtaran, E.S., Soygur, H., et al. (2007). Screening for psychological distress in Turkish cancer patients. Psycho-Oncology, 16(4), 304-311. Available from http:// onlinelibrary.wiley.com/doi/10.1002/pon.1059/pdf.

Ryan, D.A., Gallagher, P., Ryan, S.W., et al. (2012). Sensitivity and specificity of the Distress Thermometer and a two-item depression screen (Patient Health Questionnaire-2) with a "help" question for psychological distress and psychiatric morbidity in patients with advanced cancer. Psycho-Oncology, 21(12), 1275-1284. Epub ahead of print Sep 15, 2011. Available from http://onlinelibrary. wiley.com/wol1/doi/10.1002/pon.2042/full.

Sharp, L., Carsin, A.E. \& Timmons, A. (2013). Associations between cancer-related financial stress and strain and psychological well-being among individuals living with cancer. Psycho-Oncology, 22(4), 745-755. Epub ahead of print Mar 12, 2012. Available from http://onlinelibrary.wiley.com/wol1/doi/10.1002/pon.3055/full.

Shim, E.J., Shin, Y.W., Jeon, H.J., et al. (2008). Distress and its correlates in Korean cancer patients: Pilot use of the Distress Thermometer and the Problem List. PsychoOncology, 17(6), 548-555. Available from http://onlinelibrary.wiley.com/doi/10.1002/pon.1275/pdf.

Shimizu, K., Akechi, T. \& Okamura, M. (2005). Usefulness of the nurse-assisted screening and psychiatric referral program. Cancer, 103(9), 1949-1956. Available from http://onlinelibrary.wiley.com/doi/10.1002/cncr. $20992 /$ full.

Shimizu, K., Ishibashi, Y., Umezawa, S., et al. (2010). Feasibility and usefulness of the distress screening program in ambulatory care in clinical oncology practice. PsychoOncology, 19(7), 718-725. Available from http://onlinelibrary.wiley.com/doi/10.1002/pon.1616/pdf.

Söllner, W., Maislinger, S., König, A., et al. (2004). Providing psychosocial support for breast cancer patients based on screening for distress within a consultation-liaison service. Psycho-Oncology, 13(12), 893-897. Available from http://onlinelibrary.wiley.com/doi/10.1002/ pon. $867 /$ pdf.

Stefanek, M.E., Derogatis, L.R. \& Shaw, A. (1987). Psychosocial distress among oncology outpatients. Psychosomatics, 28, 530-539.

Tanaka, K., Akechi, T., Okuyama, T., et al. (2002). Impact of dyspnea, pain, and fatigue on daily life activities in ambulatory patients with advanced lung cancer. Journal of Pain and Symptom Management, 23(5), 417-423.

Tang, S.T. \& Lee, S.Y. (2004). Cancer diagnosis and prognosis in Taiwan: Patient preferences versus experiences. Psycho-Oncology, 13(1), 1-13.

Tuinman, M.A., Gazendam-Donofrio, S.M. \& HoekstraWeebers, J.E. (2008). Screening and referral for psychosocial distress in oncologic practice: Use of the Distress Thermometer. Cancer, 113(4), 870-878. Available from http://onlinelibrary.wiley.com/doi/10.1002/cncr. $23622 /$ full.

Uchitomi, Y., Mikami, I., Nagai, K., et al. (2003). Depression and psychological distress in patients during the year after curative resection of non-small-cell lung cancer. Journal of Clinical Oncology, 21(1), 69-77.

Wang, G.L., Yang, S.J., Chiu, C.Y., et al. (2009). Self-selection for psychosocial intervention by patients at a cancer center screened by Distress Thermometer as the " 6 th vital sign." Psycho-Oncology, 18(Suppl. 2), S239.

Wang, G.L., Hsu, S.H., Feng, A.C., et al. (2011). The HADS and the DT for screening psychosocial distress 
of cancer patients in Taiwan. Psycho-Oncology, 20(6), 639-646. Epub ahead of print Mar 15. Available from http://onlinelibrary.wiley.com/doi/10.1002/pon. $1952 /$ full.

Wang, Y., Zou, L., Jiang, M., et al. (2013). Measurement of distress in Chinese inpatients with lymphoma. PsychoOncology, 22(7), 1581-1586. Epub ahead of print Aug
31, 2012. Available from http://onlinelibrary.wiley. com/wol1/doi/10.1002/pon.3170/full.

Watson, M. \& Kissane, D.W. (2011). Handbook of psychotherapy in cancer care. West Sussex: Wiley-Blackwell.

Zabora, J., Brintzenhofe-Szoc, K., Curbow, B., et al. (2001). The prevalence of psychological distress by cancer site. Psycho-Oncology, 10(1), 19-28. 